\title{
A prospective case-control study comparing optical coherence tomography characteristics in neuromyelitis optica spectrum disorder- optic neuritis and idiopathic optic neuritis
}

\author{
Xiujuan Zhao', Wei Qiu², Yuxin Zhang ${ }^{1}$, Yan Luo ${ }^{1}$, Xiulan Zhang ${ }^{1}$, Lin Lu ${ }^{1 *}$ and Hui Yang ${ }^{1 *}$
}

\begin{abstract}
Background: Neuromyelitis optica spectrum disorder-optic neuritis (NMOSD-ON) can now be distinguished from other types of ON as a specific disease by the Aquaporin-4 antibody (AQP4-Ab) test. NMOSD-ON can cause severe retinal nerve fiber layer (RNFL) damage. The optical coherence tomography (OCT) characteristics between NMOSD- ON and idiopathic optic neuritis (IDON) were seldom studied in Asians.
\end{abstract}

Methods: This prospective case-control study involved 152 eyes from 143 optic neuritis (ON) patients. All the patients were divided into either the NMOSD-ON group or the IDON group based on the AQP4-Ab test. The retinal nerve fiber layer thickness (RNFLT), retinal thickness (RT), and choroidal thickness (CT) were measured by spectral-domain OCT and compared to the 60 age- and gender-matched healthy controls. The association between RNFLT and best corrected visual acuity (BCVA) was examined.

Results: The RNFLT was significantly thinner in all ON patients than in healthy controls, while NMOSD-ON eyes were significantly more affected than IDON eyes in all quadrants $(p<0.01)$. NMOSD-ON patients had stronger visual function impairment than IDON patients $(p<0.01)$. RNFLT was related to BCVA in both the NMOSD-ON and IDON groups. Microcystic macular edema (MME) was identified in 28 patients (19.58\%) and in 29 of 152 eyes (19.08\%), including 20 of 40 eyes (50\%) previously affected by ON. MME was more common in patients with NMOSD-ON (32.2\%) than in those with IDON (10.75\%) $(p=0.001)$.

Conclusions: The NMOSD-ON group had more pronounced RNFLT thinning and visual function impairment than the IDON group. MME prevalence was higher in NMOSD-ON and was associated with higher frequency of clinical relapses.

Keywords: Optical coherence tomography, Optic neuritis, Retinal nerve fiber layer, Neuromyelitis optica

\section{Background}

Optic neuritis $(\mathrm{ON})$ is an inflammatory demyelinating disease that involves the optic nerve and causes acute or subacute onset of vision loss [1]. ON is commonly involved in multiple sclerosis (MS), neuromyelitis optica (NMO), and other autoimmune diseases [2]. NMO spectrum

\footnotetext{
* Correspondence: Iulin888@126.com; 13710584767@163.com

${ }^{1}$ State Key Laboratory of Ophthalmology, Zhongshan Ophthalmic Center,

Sun Yat-Sen University, No. 54 Xianlie South Road, Guangzhou 510060,

People's Republic of China

Full list of author information is available at the end of the article
}

disorder-optic neuritis (NMOSD-ON) is one of the common types of $\mathrm{ON}$ in Asian. With the finding of aquaporin-4 antibody (AQP4-Ab) [3], NMOSD-ON can now be distinguished from other types of ON as a specific disease [4], as it is present in most patients with NMOSD [5, 6]. What's more, in 2015, the International Panel for NMO Diagnosis (IPND) achieved consensus that $\mathrm{ON}$ with AQP4-Ab seropositivity can be diagnosed as NMOSD [7] and, therefore, ON related to NMOSD was named NMOSD-ON.

Since the definition of NMOSD, NMOSD-ON has been found to differ from other types of $\mathrm{ON}$ in many 
ways. In terms of pathogenesis, NMOSD is characterized by astrocytopathy with demyelination as a secondary involvement, while MS is primarily a demyelinating disease [5]. In laboratory exams, NMOSD differs from MS in serum and cerebrospinal fluid (CSF) examination [8]. For example, NMOSD had more coexisting autoimmunity [9]. Clinically, just as we have reported before [10] and as in many other reports [11, 12] NMOSD-ON has more female preponderance, more bilateral involvement, higher relapse rate, and worse visual prognosis than IDON [6].

Spectral-domain optical coherence tomography (SD-OCT) has been used extensively in $\mathrm{ON}$ for quantifying axon damage [13]. Although there have been many reports about OCT results in MS and NMO non-ON eyes [14], the OCT characteristics of $\mathrm{ON}$ eyes in Asians are rare. After an acute ON attack in NMOSD-ON and IDON, the OCT features are not so clear. This is especially true for NMOSD-ON, because the concept of NMOSD was only defined recently. As OCT improves in data acquisition speed, resolution and reproducibility [15], different layers in the retina as well as some subtle abnormalities, such as microcystic macular edema (MME), can be more clearly recognized. With the development of enhanced depth imaging (EDI) mode scan in OCT, choroidal thickness (CT) can be accurately measured. All of these advancements broaden the characteristics that can be compared between NMOSD-ON and IDON and improve our understanding about the differences between them. Ethnicity is significant for different types of ON. In Asian populations, the incidence of NMOSD-ON is much higher than reports from Caucasian populations [10]. Although there have been numerous studies about thinner retinal nerve fiber layer thickness (RNFLT), there was a higher incidence of MME in NMO than in MS $[16,17]$. Due to the restricted availability of the AQP4-Ab test, research about the OCT characteristics of NMOSD-ON in Asian populations was rare.

To understand the influence of an acute ON attack on the retina and choroid, we analyzed the OCT characteristics six months after acute attacks in a Chinese cohort with NMOSD-ON or IDON, along with healthy controls. The OCT changes of the optic nerve and retinal and $\mathrm{CT}$ were then analyzed.

\section{Methods}

Patients with $\mathrm{ON}$ were recruited from the neuro-ophthalmology department of Zhongshan Ophthalmic Center. Recruitment took place from November 2013 to July 2015 , and patients meeting the inclusion criteria in accordance with the optic neuritis treatment trial (ONTT) [18] with first or relapsing ON were offered participation in this study. Exclusion criteria included any of the following: any evidence of toxic, vascular, infiltrative, compressive, metabolic, hereditary optic neuropathy, causative ocular diseases or retinal lesions [18], fulfilled the diagnostic criteria of MS McDonald's criteria [19].

Exclusion criteria also comprised an intraocular pressure higher than $21 \mathrm{mmHg}$, prior ocular trauma, systemic conditions that could affect the visual function, a significant refractive error more than $3 \mathrm{D}$ of spherical equivalent refraction or 2D of astigmatism, a history of glaucoma, retinal disease, laser therapy or media opacification. The data were analyzed after the patients had an episode of ON more than six months when the visual functional and structural changes were stabilized. Exclusion criteria also applied to the controls as for ON patients.

The patients were divided into three groups: NMOSDON, IDON, and healthy controls. NMOSD-ON included patients who met the established diagnostic criteria for NMO or NMOSD published by Wingerchuk et al. [7]. IDON group patients included those with typical acute demyelinating $\mathrm{ON}$ and AQP4-Ab seronegative, none of patients with AQP4-ab negative fullfiled NMOSD criteria or McDonald MS criteria [19].

This study complied with informed consent regulations and the Declaration of Helsinki. A verbal informed consent was needed before subject enrollment in the study.

Laboratory and radiological results were recorded. Serum was drawn for extractable nuclear antigen antibodies (SSA/SSB), antinuclear antibody (ANA), rheumatoid factor (RF), anti-double standard deoxyribonucleic acid (anti-ds DNA), anti-cardiolipin antibodies (ACLs), and AQP4-Ab at the Third Affiliated Hospital of Sun Yat-sen University. All serum samples were analyzed for the presence of AQP4-Ab by indirect immunofluorescence using human AQP4-transfected cells from a commercial BIOCHIP kit (Euroimmun, Germany) as described previously [20]. Clinical data was recorded along with the AQP4-Ab. Patients were sub-divided into the NMOSD-ON group or the IDON group according to the results of the AQP4-Ab test. All patients were treated with corticosteroids.

RNFLT was obtained on a high-definition spectral-domain optical coherence tomography (HD-OCT) with EDI mode (Heidelberg Engineering, Heidelberg, Germany). Measurements were taken using the Spectralis $3.5 \mathrm{~mm}$ standard circle scan protocol with version 5.3 software, with signal strength greater than 21 . The RNFLT around the optic nerve head in a circle with a minimum of 50 automatic real time (ART) and yields a temporal, superior, nasal, inferior and mean overall graph. Nasal-to-temporal RNFL ratio (N/T ratio) were performed in all patients. A macular scan consisting of 25 horizontal scans centered on the fovea was performed. The central foveal thickness values were automatically generated by the imaging software. CT was defined as the distance between the retinal pigment epithelium (RPE) and the outer border of choroid 
at the subfoveal location. All OCT measurements were manually performed by one observer who was blinded with the clinical diagnosis and was not involved in the data analysis. MME [21-23] was defined as cystic, honeycombed, lacunar area of hyporeflectivity with clear boundaries in two or more consecutive SD-OCT macular raster scan images [24] (Fig. 1). The BCVA was converted to the logarithm of the minimum angle of resolution ( $\log M A R)$ for statistical analysis.

\section{Statistics}

All of the data was analyzed using SPSS 19.0 (SPSS Inc., Chicago, IL, USA). First, we determined the mean value (presented as mean \pm standard deviation) of RNFLT, central foveal thickness, and CT before we used the one-way ANOVA test and Bonferroni adjustment to compare multiple variables between the different groups. Associations between BCVA and RNFLT were evaluated using the univariate linear regression. All $p$ values were two-sided and statistical significance was established at $p<0.05$.

\section{Outcome measures}

The primary outcome measures were RNFLT compared in NMOSD-ON, IDON patients, and healthy controls. Secondary outcome measures included MME, central foveal thickness, CT, correlations between RNFLT and BCVA.
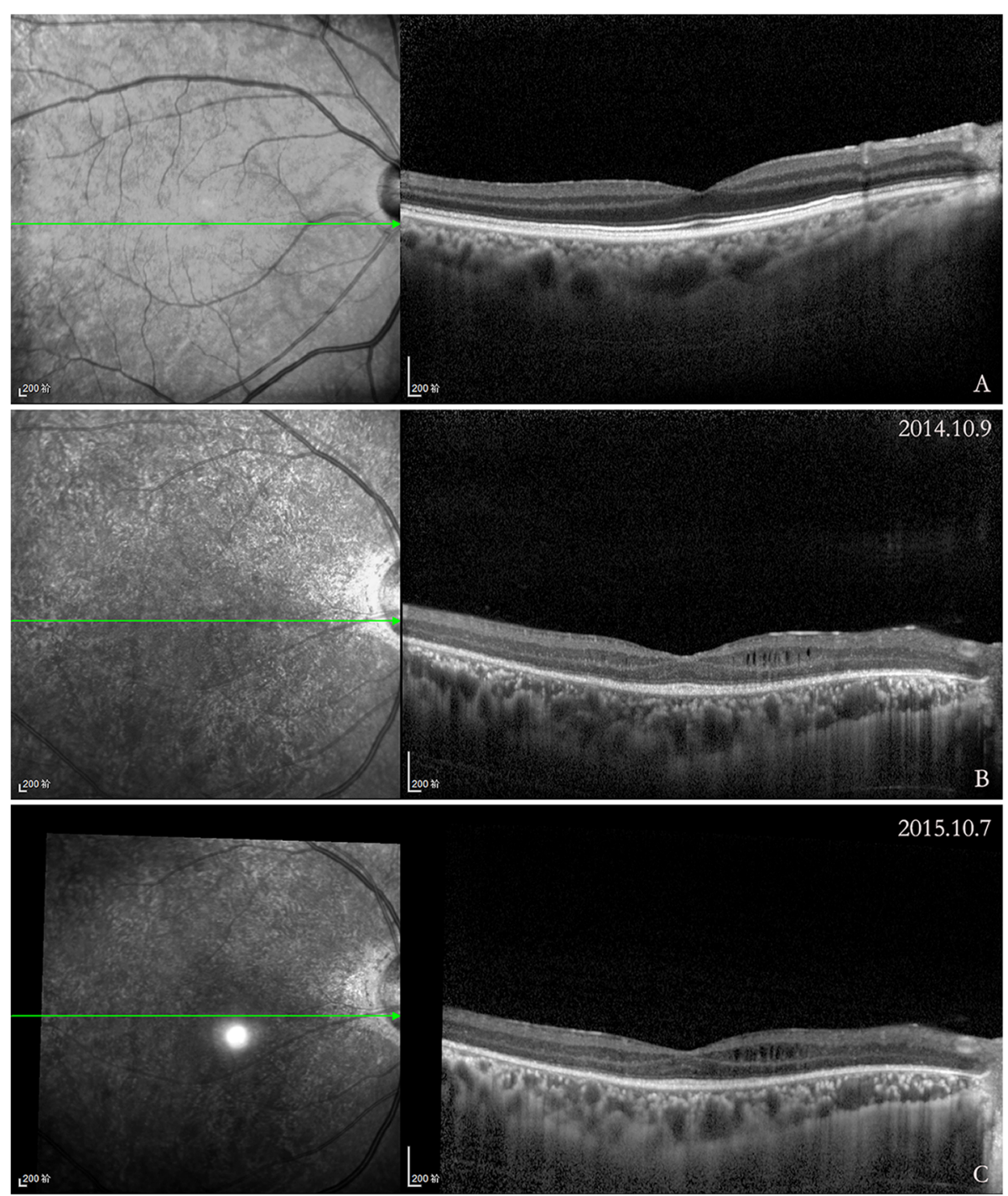

Fig. 1 Scans of a NMOSD-ON eyes with and without microcystic macular edema (MME). On the left, it is the infrared fundus image and the green line marks the position of the corresponding OCT image on the right. $\mathbf{a}$, Macular scan of NMOSD-ON eye. $\mathbf{b}$, MME of the inner nuclear layer on OCT in NMOSD-ON eye. c. At one year follow-up, the MME was increased (white arrow) 


\section{Results}

A total of 143 patients were evaluated; among them, 59 eyes (52 patients) were in the NMOSD-ON group and 93 eyes (91 patients) were in the IDON group. All NMOSD-ON patients were AQP4-Ab positive. The demographic data of the study cohort is summarized in Table 1.

\section{OCT measures in ON patients}

Overall RNFLT and four quadrants RNFLT were significantly thinner in both NMOSD-ON and IDON eyes compared to healthy eyes, with NMOSD-ON eyes being more affected than IDON eyes significantly $(p<0.01)$ (Fig. 2). Central foveal thickness trended higher in ON eyes than healthy eyes, and NMOSD-ON eyes higher than IDON eyes $(p<0.05)$. The subfoveal CT in ON eyes was not different from the healthy controls $(p>0.05)$ (Fig. 3). In clinic work, a temporal preponderance was often seen in optic atrophy with ON and OCT showed particular damage to temporal axons $[25,26]$. While peripapillary RNFLT (pRNFLT) was primarily thinned in the temporal quadrant in both NMOSD-ON and IDON eyes, pRNFLT of other quadrants in NMOSD-ON eyes was much more reduced than IDON eyes. In order to identify whether a predilection of the temporal quadrant of pRNFL existed, we used the $\mathrm{N} / \mathrm{T}$ ratio to compare quadrant thinning in the groups of NMOSD-ON and IDON patients [27], but the difference was not statistically significant $(0.96 \pm 0.43$ vs. $1.01 \pm 0.46 ; \mathrm{F}=0.298, p=0.586)$. NMOSD-ON eyes with a history of one $\mathrm{ON}$ event were different from IDON eyes with one ON event $(p<0.01)$. NMOSD-ON eyes with more than one $\mathrm{ON}$ event were not different from MS-ON eyes with more than one ON event and NMOSD-ON eyes with one $\mathrm{ON}$ event $(p>0.05)$ (Table 2$)$. Both in NMOSD-ON and IDON eyes, BCVA is strongly correlated with overall and four quadrants RNFLT $(p<0.05)$ (Table 3).

\section{Role of microcystic macular edema (MME)}

MME was identified on OCT in 28 patients (19.58\%), and in 29 of 152 eyes (19.08\%), including 20 of 40 eyes

Table 1 Demographic data of NMOSD-ON and IDON patients

\begin{tabular}{|c|c|c|c|}
\hline Baseline characteristics & NMOSD-ON $(n=52)$ & IDON $(n=91)$ & $P$ \\
\hline Age at first clinical attack, y, median (IQR) & $35.00(20.75-47.00)$ & $37.00(27.00-49.00)$ & $0.427^{a}$ \\
\hline Age at serum sampling, y, median (IQR) & $36.50(21.00-47.00)$ & $37.00(28.00-50.00)$ & $0.196^{a}$ \\
\hline Sex, F:M & $23: 3$ & $50: 41$ & $0.000^{\mathrm{b}}$ \\
\hline No. of patients with recurrent ON & 26 & 14 & $0.000^{\mathrm{b}}$ \\
\hline No. of patients with ION & 26 & 77 & $0.000^{\mathrm{b}}$ \\
\hline No. of ON episodes, mean \pm SD & $1.58 \pm 1.54$ & $1.04 \pm 0.19$ & $0.007^{\mathrm{a}}$ \\
\hline Interval relapsing time, m, median (IQR) & $12.00(6.00-48.00)$ & $36.00(20.25-63.00)$ & $0.237^{\mathrm{a}}$ \\
\hline Interval from onset of last ON attack to OCT test, $m$, mean \pm SD & $6.72 \pm 1.32$ & $6.31 \pm 1.43$ & $0.531^{a}$ \\
\hline Visual acuity at baseline (logMAR), mean \pm SD & $2.17 \pm 1.38$ & $1.49 \pm 1.23$ & $0.044^{a}$ \\
\hline ON event with visual acuity worse than $1.0 \log M A R(n, \%)$ & $43,82.69 \%$ & $49,53.85 \%$ & $0.001^{\mathrm{b}}$ \\
\hline At least one episode with no light perception (n, \%) & $13,25.00 \%$ & $8,8.79 \%$ & $0.008^{b}$ \\
\hline Abnormal brain MRI (n, \%) & $9,17.31 \%$ & $5,5.49 \%$ & $0.027^{b}$ \\
\hline Blood test & 37 & 53 & $0.50^{c}$ \\
\hline SSA $(n, \%)$ & $3,8.11 \%$ & $3,5.66 \%$ & \\
\hline Anti-ds DNA (n, \%) & $3,8.11 \%$ & $1,1.89 \%$ & \\
\hline $\mathrm{ACL}(\mathrm{n}, \%)$ & $1,2.70 \%$ & $1,1.89 \%$ & \\
\hline Accelerated ESR (n, \%) & $3,8.11 \%$ & $1,1.89 \%$ & \\
\hline Increased CRP (n, \%) & $2,5.41 \%$ & $7,13.21 \%$ & \\
\hline ANCA positive (n, \%) & $2,5.41 \%$ & $2,3.77 \%$ & \\
\hline ANA ( $\geq 1: 320, \%)$ & $9,24.32 \%$ & $6,11.32 \%$ & \\
\hline \multicolumn{4}{|l|}{ Follow-up outcomes } \\
\hline No. of cases & 48 & 85 & \\
\hline Visual acuity at last follow-up (logMAR), mean \pm SD & $1.35 \pm 1.30$ & $0.77 \pm 0.97$ & $0.001^{a}$ \\
\hline Cases with myelitis episodes (n, \%) & $12,23.08 \%$ & $1,1.10 \%$ & $0.000^{\mathrm{b}}$ \\
\hline
\end{tabular}

NMOSD-ON neuromyelitis optica spectrum disorder- optic neuritis, IDON idiopathic optic neuritis, IQR interquartile range, ION isolated optic neuritis, SSA anti-Ro/ SSA antibody, anti-ds DNA anti-double standard deoxyribonucleic acid, $A C L$ anti-cardiolipin antibody, ESR erythrocyte sedimentation rate, CRP C-reactive protein, ANCA antineutrophil cytoplasmic antibody, ANA antinuclear antibody, ${ }^{\mathrm{a}}$ Mann-Whitney $\mathrm{U}$ test, ${ }^{\mathrm{b}} \mathrm{Chi}$-squared test, ${ }^{\mathrm{C}}$ Fisher's exact test 


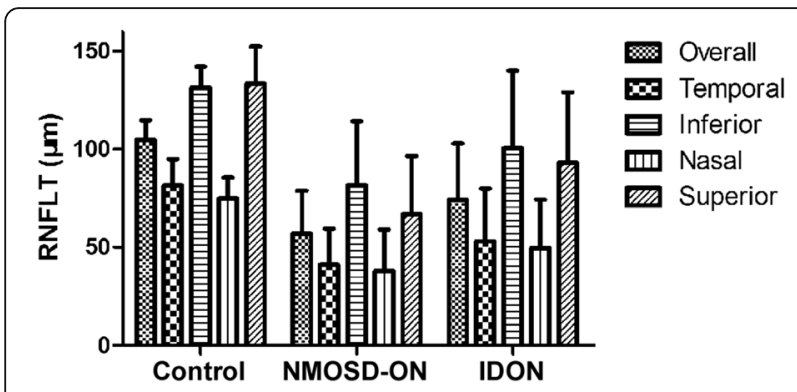

Fig. 2 Comparisons of Overall and quadrants RNFLT among control, NMOSD-ON and IDON. Overall and four quadrants RNFLT were significantly thinner in both NMOSD-ON and IDON eyes compared to healthy eyes, with NMOSD-ON eyes being more thinner than IDON eyes significantly $(p<0.01)$. RNFLT: retinal nerve fiber layer thickness; NMOSD-ON: neuromyelitis optica spectrum disorder- optic neuritis; IDON: idiopathic optic neuritis

(50\%) with more than one episode of ON. MME was more common in patients with NMOSD-ON $(32.20 \%$, 19 of 59 eyes) than in those with IDON (10.75\%, 10 of 93 eyes) $(p=0.001)$. A total of $69.0 \%$ of eyes with MME had prior ON. There were no appreciable differences in age or sex between patients with and without MME. Eyes with MME had lower vision, but the difference did not reach statistical significance. The overall RNFLT was $19.23 \mu \mathrm{m}$ thinner in eyes with MME compared to all ON eyes without MME $(p=0.03)$. The overall RNFLT was $5.39 \mu \mathrm{m}$ lower in eyes with MME compared to ON eyes with prior ON without MME $(p=0.36)$. Central foveal thickness was $10.42 \mu \mathrm{m}$ higher in eyes with MME compared with eyes without MME $(p=0.18)$.

\section{Discussion}

In this study, a prospective case-control study was made to compare the OCT characteristics between NMOSD-ON and IDON. The study had a large NMOSD-ON sample size and the comparison was not limited to RNFLT, but also included macular and choroidal thicknesses.

As for the clinical features of these patients, there was a strong female predominance, higher recurrent $\mathrm{ON}$ frequency, and more severe visual function damage in the NMOSD-ON group.

RNFLT was significantly thinner in NMOSD-ON and IDON patients six months after an ON attack compared to normal controls. It was reported that NMOSD-ON had no preponderant RNFLT thinning pattern [28]. In this study, no significant N/T ratio difference was found in NMOSD-ON versus IDON eyes, which means all quadrants were evenly affected in both types of ON. This was consistent with previous studies from the Asian cohort, which also failed to demonstrate the similar pattern [29]. On the other hand, the severity of the ON studied might also influence the RNFLT pattern. The temporal RNFLT contains the papillomacular bundle, which is more vulnerable to pathologic damage. When the ON attack was not as severe, quadrants other than the temporal might be relatively damaged. Further investigation is needed to identify whether there were indeed pattern differences or ethnic differences in the pathologic involvement of the optic nerve and severity of the ON attack.

Damage of the optic nerve from a single ON attack was more severe in NMOSD patients. RNFLT became much thinner in the NMOSD-ON group than IDON group, which was in line with previous OCT studies [30]. What's more, this study also demonstrated that RNFLT thinning in first-ever ON was significantly more severe in NMOSD-ON eyes than in IDON eyes. Ratchford et al. [31] suggested that RNFLT differences of more than $15 \mu \mathrm{m}$ between eyes after a first episode of unilateral $\mathrm{ON}$ should prompt consideration of an NMOSD-ON. No significant difference in RNFLT thinning could be found between NMOSD-ON eyes with first-ever $\mathrm{ON}$ and those with recurrent $\mathrm{ON}$ (RON), which indicated that a single NMOSD-ON attack might be severe enough to destroy most of the RNFLT. This also implied that a functional index, such as visual field or visual evoked potentials, would be more suitable for evaluating the severity of visual damage in RON than RNFLT.

BCVA is strongly correlated with RNFLT, not only with average RNFLT, but also with all quadrants of

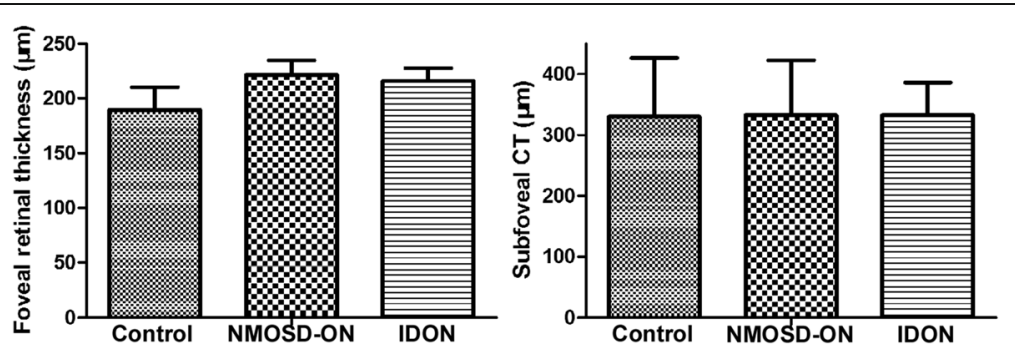

Fig. 3 Comparisons of foveal retinal thickness and subfoveal choroidal thickness among control, NMOSD-ON and IDON. Foveal retinal thickness was higher in ON eyes than healthy eyes, and NMOSD-ON eyes higher than IDON eyes $(p<0.05)$. The subfoveal CT in ON eyes was not different from the healthy controls $(p>0.05)$ 
Table 2 Statistical analysis of overall RNFLT for optic neuritis patients and controls

\begin{tabular}{lll}
\hline Overall RNFLT & & $P$ values \\
\hline $\begin{array}{l}\text { NMOSD-ON with 1 ON } \\
(n=32)\end{array}$ & Healthy controls $(n=60)$ & 0.000 \\
$59.67 \pm 22.19$ & $105.81 \pm 10.60$ & \\
IDON with 1 ON $(n=79)$ & Healthy controls $(n=60)$ & 0.000 \\
$75.48 \pm 28.15$ & $105.81 \pm 10.60$ & \\
NMOSD-ON with 1 ON & IDON with 1 ON $(n=79)$ & 0.001 \\
$(n=32)$ & & \\
$59.67 \pm 22.19$ & $75.48 \pm 28.15$ & \\
NMOSD-ON with $>1$ ON & IDON with $>1$ ON $(n=14)$ & 0.644 \\
$(n=27)$ & & \\
$45.44 \pm 16.03$ & $51.80 \pm 32.51$ & 0.120 \\
$\begin{array}{l}\text { NMOSD-ON with 1 ON } \\
(n=32)\end{array}$ & $\begin{array}{l}\text { NMOSD-ON with }>1 \text { ON } \\
(n=27)\end{array}$ \\
$59.67 \pm 22.19$ & $45.44 \pm 16.03$ & \\
IDON with 1 ON $(n=79)$ & IDON with $>1$ ON $(n=14)$ & 0.038 \\
$75.48 \pm 28.15$ & $51.80 \pm 32.51$ &
\end{tabular}

RNFLT retinal nerve fiber layer thickness, ON optic neuritis, NMOSD-ON neuromyelitis optica spectrum disorder- optic neuritis, IDON idiopathic optic neuritis. Wilcoxon Rank Sum Tests with Holm Correction

RNFLT in both NMOSD-ON and IDON eyes. This is in accordance with values reported by earlier studies [29, 32]. Schneider, E., et al. [27] reported that NMOSD-ON eyes showed more apparent association of structural retinal damage and impairment of visual function than in MS-ON eyes. The possible reason may be that half of the NMOSD-ON eyes had pRNFLT below $46.6 \mu \mathrm{m}$ versus none of the IDON eyes in their study. In our study, there were 19 NMOSD-ON eyes and 17 IDON eyes with pRNFLT below $47 \mu \mathrm{m}$. The thinner the RNFLT becomes, the stronger the association between morphology and BCVA becomes. We suggest this might be due to the fact that visual function is no longer able to be maintained after RNFLT decreases to a certain threshold [29, 31].

As for retinal structures, the central foveal thicknesses of both groups were thicker than healthy controls, and

Table 3 Correlation between BCVA and RNFLT using univariate linear regression

\begin{tabular}{llllll}
\hline \multirow{2}{*}{ RNFLT } & \multicolumn{2}{l}{ NMOSD-ON } & & & \multicolumn{2}{l}{ IDON } \\
\cline { 2 - 3 } & \multicolumn{1}{l}{ BCVA } & & & BCVA & \\
\cline { 2 - 3 } & $B$ & & & & \\
\hline Global & -4.824 & 0.031 & & -2.425 & 0.042 \\
Superior & -7.769 & 0.026 & & -1.465 & 0.028 \\
Nasal & -5.173 & 0.034 & & -1.376 & 0.036 \\
Inferior & -8.435 & 0.031 & & -1.168 & 0.033 \\
Temporal & -4.907 & 0.044 & -2.084 & 0.047 \\
\hline
\end{tabular}

NMOSD-ON neuromyelitis optica spectrum disorder- optic neuritis, IDON idiopathic optic neuritis, $B C V A$ best corrected visual acuity, RNFLT retinal nerve fiber layer thickness
NMOSD-ON eyes were thicker than IDON eyes. The differences were primarily driven by MME, which caused a thickening of the inner nuclear layer and outer retinal layers [27]. MME could be a manifestation of inner nuclear layer (INL) pathology. Both Sotirchos et al. [17] and Gelfand et al. [24] reported MME existed in NMO eyes affected by ON. Also, it can be seen in 5-6\% of MS patients [6]. MME was associated with more severe MS and poorer VA [33]. In this study, MME was found in $32.2 \%$ of NMOSD-ON patients and $10.75 \%$ of IDON patients, but not in the healthy control. We also found that MME was associated with more severe RNFLT thinning and more profoundly impaired BCVA. MME occurred in various neuroinflammatory disorders associated with ON [34]. MME might be linked to Müller cell pathology [35], which is caused by AQP4-Ab via a leaky blood-retina barrier. Also, there might be a pathophysiological correlation between the extent of damage to the optic nerve and MME. The findings in this study showed that eyes with more severe $\mathrm{ON}$ had a higher incidence of MME.

CT is an index of eye circulation. The concentration of AQP4-Ab in serum was much higher than that in CSF [36-38] and there were reports of retina blood vessel abnormality in NMO [39]. All of these indicating mechanisms of retina blood barrier (RBB) and blood brain barrier (BBB) damage might be involved in the pathogenesis of NMO. Whether different types of ON have an effect on choroidal vessel structure has not yet been studied. Ebru Esen et al. [40] reported that the mean subfoveal CT was reduced significantly in MS patients versus the healthy controls. However, in this study, there was no significant difference between IDON and the normal controls, or between NMOSD-ON and IDON. Diurnal variations of CT [41] could have an effect on the results, because the data was not obtained at the same time of the day. In our study, the number of subfoveal CTs in the normal controls was in accordance with other reports $[42,43]$ while CT of the healthy control in Ebru Esen et al.'s study was much thicker [40]. Determining whether choroidal vessels are involved in the pathology of the disease requires a larger cohort and more detailed measurements.

Using OCT for differentiation of IDON from NMOSD$\mathrm{ON}$ has long been desirable. However, so far, OCT alone has not been enough for differentiation, though OCT shows a promising effect in investigating the pathologic difference, managing the severity of $\mathrm{ON}$, and evaluating the therapeutic effect on ON [44]. Still, there were some limitations to this study. The relationship between RNFLT change and visual function remains to be investigated. Multilayer segmentation of greater detail should be analyzed. Anti-myelin oligodendrocyte glycoprotein (MOG) antibodies are frequently associated with the recurrent 
ON/chronic relapsing inflammatory ON phenotype, which is highly sensitive to even low doses of oral corticosteroids [45]. There is still a debate about whether MOG-Ab positive patients will be considered part of the NMOSD, or rather a distinct disease entity [46]. We did not test the anti-MOG antibody in AQP4-Ab negative patients in our study, which may somewhat skew the results. However, this could provide a direction for future research.

\section{Conclusion}

In summary, patients with NMOSD-ON had more pronounced RNFLT thinning than patients with IDON and was closely associated with visual function impairment.

\section{Abbreviations}

ACLs: Anti-cardiolipin antibodies; ANA: Antinuclear antibody; anti-ds DNA: anti-double standard deoxyribonucleic acid; AQP4-Ab: Aquaporin-4 antibody; BCVA: Best corrected visual acuity; CIS: Clinically isolated syndrome; CSF: Cerebrospinal fluid; CT: Choroidal thickness; EDI: Enhanced depth imaging; IDON: Idiopathic optic neuritis; MME: Microcystic macular edema; MS: Multiple sclerosis; MS-ON: MS-related optic neuritis; NMOSD: Neuromyelitis optica spectrum disorder; NMOSD-ON: NMOSD-related optic neuritis; OCT: Optical coherence tomography; ONTT: Optic neuritis treatment trial; RF: Rheumatoid factor; RNFT: Retinal nerve fiber layer thickness; RPE: Retinal pigment epithelium

\section{Funding}

National Basic Research Development Program of China (973 program: 2013CB967000), the National Natural Science Foundation of China to LIN LU (81570862), YAN LUO (81371020), the Natural Science Foundation of Guangdong Province of China to HUI YANG (S2012010008439).

\section{Availability of data and materials}

The datasets used and analyzed during the current study are available from the corresponding author on reasonable request

\section{Authors' contributions}

HY and LL conceived the study, coordinated its design and drafted the manuscript. WQ and YL had significant input into study protocol. XJZ and XLZ were involved in critical appraisal and revision of the manuscript. YXZ provided statistical expertise. All authors read and approved the final manuscript.

\section{Ethics approval and consent to participate}

A verbal informed consent was obtained from all of the included subjects.

\section{Consent for publication}

Not applicable for this study.

\section{Competing interests}

The authors declare that they have no competing interest.

\section{Publisher's Note}

Springer Nature remains neutral with regard to jurisdictional claims in published maps and institutional affiliations.

\footnotetext{
Author details

${ }^{1}$ State Key Laboratory of Ophthalmology, Zhongshan Ophthalmic Center, Sun Yat-Sen University, No. 54 Xianlie South Road, Guangzhou 510060 People's Republic of China. 'Department of Neurology, The Third Affiliated Hospital of Sun Yat-Sen University, Guangzhou, China.
}

Received: 11 October 2017 Accepted: 28 August 2018

Published online: 14 September 2018

\section{References}

1. Beck RW, Cleary PA, Anderson MM Jr, et al. A randomized, controlled trial of corticosteroids in the treatment of acute optic neuritis. The optic neuritis study group. N Engl J Med. 1992;326(9):581-8.
2. Petzold A, Wattjes MP, Costello F, et al. The investigation of acute optic neuritis: a review and proposed protocol. Nat Rev Neurol. 2014;10(8):447-58.

3. Zekeridou A, Lennon VA. Aquaporin-4 autoimmunity. Neurol Neuroimmunol Neuroinflamm. 2015:2(4):e110.

4. Metz I, Beissbarth T, Ellenberger D, et al. Serum peptide reactivities may distinguish neuromyelitis optica subgroups and multiple sclerosis. Neurol Neuroimmunol Neuroinflamm. 2016;3(2):e204.

5. Lennon VA, Wingerchuk DM, Kryzer TJ, et al. A serum autoantibody marker of neuromyelitis optica: distinction from multiple sclerosis. Lancet. 2004; 364(9451):2106-12.

6. Bennett JL, de Seze J, Lana-Peixoto M, et al. Neuromyelitis optica and multiple sclerosis: seeing differences through optical coherence tomography. Mult Scler. 2015;21(6):678-88.

7. Wingerchuk DM, Banwell B, Bennett JL, et al. International consensus diagnostic criteria for neuromyelitis optica spectrum disorders. Neurology. 2015;85(2):177-89.

8. Jarius S, Paul F, Franciotta D, et al. Cerebrospinal fluid findings in aquaporin4 antibody positive neuromyelitis optica: results from 211 lumbar punctures. J Neurol Sci. 2011:306(1-2):82-90.

9. Jarius S, Ruprecht K, Wildemann B, et al. Contrasting disease patterns in seropositive and seronegative neuromyelitis optica: a multicentre study of 175 patients. J Neuroinflammation. 2012;9:14.

10. Yang H, Qiu W, Zhao X, et al. The correlation between Aquaporin-4 antibody and the visual function of patients with demyelinating optic neuritis at onset. J Ophthalmol. 2015;2015:672931.

11. Kitley J, Leite MI, Nakashima I, et al. Prognostic factors and disease course in aquaporin-4 antibody-positive patients with neuromyelitis optica spectrum disorder from the United Kingdom and Japan. Brain. 2012;135(Pt 6):1834-49.

12. Merle $\mathrm{H}$, Olindo S, Bonnan $\mathrm{M}$, et al. Natural history of the visual impairment of relapsing neuromyelitis optica. Ophthalmology. 2007;114(4):810-5.

13. Pulicken M, Gordon-Lipkin E, Balcer LJ, et al. Optical coherence tomography and disease subtype in multiple sclerosis. Neurology. 2007:69(22):2085-92.

14. Outteryck O, Majed B, Defoort-Dhellemmes S, et al. A comparative optical coherence tomography study in neuromyelitis optica spectrum disorder and multiple sclerosis. Mult Scler. 2015;21(14):1781-93.

15. Bock M, Brandt AU, Dorr J, et al. Time domain and spectral domain optical coherence tomography in multiple sclerosis: a comparative cross-sectional study. Mult Scler. 2010;16(7):893-6.

16. Kaufhold F, Zimmermann H, Schneider E, et al. Optic neuritis is associated with inner nuclear layer thickening and microcystic macular edema independently of multiple sclerosis. PLoS One. 2013;8(8):e71145.

17. Sotirchos ES, Saidha S, Byraiah G, et al. In vivo identification of morphologic retinal abnormalities in neuromyelitis optica. Neurology. 2013;80(15):1406-14.

18. The clinical profile of optic neuritis. Experience of the Optic Neuritis Treatment Trial. Optic neuritis study group. Arch Ophthalmol. 1991;109(12):1673-8.

19. Polman CH, Reingold SC, Banwell B, et al. Diagnostic criteria for multiple sclerosis: 2010 revisions to the McDonald criteria. Ann Neurol. 2011;69(2):292-302

20. Long Y, Qiu W, Lu Z, et al. Aquaporin 4 antibodies in the cerebrospinal fluid are helpful in diagnosing Chinese patients with neuromyelitis optica. Neuroimmunomodulation. 2012;19(2):96-102.

21. Cruz-Herranz A, Balk LJ, Oberwahrenbrock T, et al. The APOSTEL recommendations for reporting quantitative optical coherence tomography studies. Neurology. 2016:86(24):2303-9.

22. Schippling S, Balk L, Costello F, et al. Quality control for retinal OCT in multiple sclerosis: validation of the OSCAR-IB criteria. Mult Scler. 2015;21(2):163-70.

23. Tewarie P, Balk L, Costello F, et al. The OSCAR-IB consensus criteria for retinal OCT quality assessment. PLoS One. 2012;7(4):e34823.

24. Gelfand JM, Cree BA, Nolan R, et al. Microcystic inner nuclear layer abnormalities and neuromyelitis optica. JAMA Neurol. 2013;70(5):629-33.

25. Bock $M$, Brandt $A U$, Dorr J, et al. Patterns of retinal nerve fiber layer loss in multiple sclerosis patients with or without optic neuritis and glaucoma patients. Clin Neurol Neurosurg. 2010;112(8):647-52.

26. Kerrison JB, Flynn T, Green WR. Retinal pathologic changes in multiple sclerosis. Retina. 1994:14(5):445-51.

27. Schneider E, Zimmermann H, Oberwahrenbrock T, et al. Optical coherence tomography reveals distinct patterns of retinal damage in Neuromyelitis Optica and multiple sclerosis. PLoS One. 2013;8(6):e66151.

28. Mateo J, Esteban $\mathrm{O}$, Martinez M et al. The contribution of optical coherence tomography in Neuromyelitis Optica Spectrum disorders. Front Neurol. 2017;8:493.

29. Trip SA, Schlottmann PG, Jones SJ, et al. Retinal nerve fiber layer axonal loss and visual dysfunction in optic neuritis. Ann Neurol. 2005;58(3):383-91. 
30. Nakamura M, Nakazawa T, Doi H, et al. Early high-dose intravenous methylprednisolone is effective in preserving retinal nerve fiber layer thickness in patients with neuromyelitis optica. Graefes Arch Clin Exp Ophthalmol. 2010;248(12):1777-85.

31. Ratchford JN, Quigg ME, Conger A, et al. Optical coherence tomography helps differentiate neuromyelitis optica and MS optic neuropathies. Neurology. 2009;73(4):302-8.

32. Fisher JB, Jacobs DA, Markowitz CE, et al. Relation of visual function to retinal nerve fiber layer thickness in multiple sclerosis. Ophthalmology. 2006;113(2):324-32.

33. Gelfand JM, Nolan R, Schwartz DM, et al. Microcystic macular oedema in multiple sclerosis is associated with disease severity. Brain. 2012;135(Pt 6):1786-93.

34. Brandt AU, Oberwahrenbrock T, Kadas EM, et al. Dynamic formation of macular microcysts independent of vitreous traction changes. Neurology. 2014;83(1):73-7.

35. Balk $\sqcup$, Killestein J, Polman $\mathrm{CH}$, et al. Microcystic macular oedema confirmed, but not specific for multiple sclerosis. Brain. 2012;135(Pt 12):e226. author reply e7

36. Jarius S, Franciotta D, Paul F, et al. Cerebrospinal fluid antibodies to aquaporin-4 in neuromyelitis optica and related disorders: frequency, origin, and diagnostic relevance. J Neuroinflammation. 2010;7:52.

37. Majed M, Fryer JP, McKeon A, et al. Clinical utility of testing AQP4-IgG in CSF: guidance for physicians. Neurol Neuroimmunol Neuroinflamm. 2016;3(3):e231.

38. Wingerchuk DM, Lucchinetti CF. Comparative immunopathogenesis of acute disseminated encephalomyelitis, neuromyelitis optica, and multiple sclerosis. Curr Opin Neurol. 2007;20(3):343-50.

39. Vincent $T$, Saikali $P$, Cayrol R, et al. Functional consequences of neuromyelitis optica-lgG astrocyte interactions on blood-brain barrier permeability and granulocyte recruitment. J Immunol. 2008;181(8):5730-7.

40. Esen E, Sizmaz S, Demir T, et al. Evaluation of Choroidal vascular changes in patients with multiple sclerosis using enhanced depth imaging optical coherence tomography. Ophthalmologica. 2016;235(2):65-71.

41. Lee SW, Yu SY, Seo KH, et al. Diurnal variation in choroidal thickness in relation to sex, axial length, and baseline choroidal thickness in healthy Korean subjects. Retina. 2014;34(2):385-93.

42. Agawa T, Miura M, Ikuno Y, et al. Choroidal thickness measurement in healthy Japanese subjects by three-dimensional high-penetration optical coherence tomography. Graefes Arch Clin Exp Ophthalmol. 201 1;249(10):1485-92.

43. Margolis R, Spaide RF. A pilot study of enhanced depth imaging optical coherence tomography of the choroid in normal eyes. Am J Ophthalmol. 2009:147(5):811-5.

44. Jacob A, Hutchinson M, Elsone L, et al. Does natalizumab therapy worsen neuromyelitis optica? Neurology. 2012;79(10):1065-6.

45. Chalmoukou K, Alexopoulos H, Akrivou S, et al. Anti-MOG antibodies are frequently associated with steroid-sensitive recurrent optic neuritis. Neurol Neuroimmunol Neuroinflamm. 2015;2(4):e131.

46. Zamvil SS, Slavin AJ. Does MOG Ig-positive AQP4-seronegative opticospinal inflammatory disease justify a diagnosis of $\mathrm{NMO}$ spectrum disorder? Neurol Neuroimmunol Neuroinflamm. 2015;2(1):e62.

Ready to submit your research? Choose BMC and benefit from:

- fast, convenient online submission

- thorough peer review by experienced researchers in your field

- rapid publication on acceptance

- support for research data, including large and complex data types

- gold Open Access which fosters wider collaboration and increased citations

- maximum visibility for your research: over $100 \mathrm{M}$ website views per year

At $\mathrm{BMC}$, research is always in progress.

Learn more biomedcentral.com/submissions 